\title{
Massive hydrothorax during supracostal approach for percutaneous nephrolithotomy: A case report
}

\author{
Anju Rani ${ }^{*}$, Pawan Kumar ${ }^{2}$ \\ ${ }^{1}$ Assistant Professor, ${ }^{2}$ Junior Resident, ${ }^{1}$ Dept. of Anaesthesia, ${ }^{2}$ Dept. of Oral \& Maxillofacial Surgery, PGIMS Rohtak, Haryana, India
}

*Corresponding Author: Anju Rani

Email: anjudahiya88@gmail.com

Received: $22^{\text {nd }}$ November, 2018

Accepted: $18^{\text {th }}$ February, 2019

\begin{abstract}
Percutaneous nephrolithotomy (PCNL) is a technique commonly used for renal stone disease. Supracostal approach is required many times for stone in upper renal calices, with significant risk of hydrothorax and pneumothorax. In this case report, we are discussing hydrothorax as a complication of PCNL performed via supracostal approach.
\end{abstract}

Keywords: Massive Hydrothorax, Supracostal approach, Percutaneous nephrolithotomy (PCNL), Pneumothorax.

\section{Introduction}

In recent years, PCNL is being increasingly used for urinary calculi. It has many advantages compared to open approach as it avoids larger incision hence decreasing postoperative morbidiy of the patients, but also associated with some technical complications. Hydrothorax and pneumothorax are common, with ARDS, cardiac arrest being among few rare complications.

\section{Case Report}

A 55 year old female having nephrolithiasis in upper calyx of right kidney, was posted for PCNL surgery. In preoperative evaluation, patient accepted for anaesthesia under ASA grade I. Pre-operative ECG and chest $\mathrm{x}$-ray was normal. Surgery was done under subarachnoid block. Adequate regional anaesthesia was achieved (T6). Patient turned to prone position. Oxygen was given via venti mask. At the end of surgery patient started complaining of difficulty in breathing, spo2 gradually dropped down to $70 \%$. Patient also became tachypneic. On chest auscultation, air entry on right side (operative side) was almost nil. Immediately patient was turned to supine position and given $100 \%$ oxygen via bain's circuit. Spo 2 improved to $95 \%$ but patient kept complaining of difficulty in breathing. Also blood pressure dropped to $85 / 42 \mathrm{mmhg}$. Pleural injury was suspected so chest $\mathrm{x}$-ray done under c-arm but unfortunately no clear image was observed. Needle aspiration was tried which lead to aspiration of fluid. Then intercostal chest drain was inserted and $1800 \mathrm{ml}$ of irrigation fluid was drained. Patient was monitored on OT table for 40 minutes. Patient was again hemodynamically stable. Gradually air entry on right side significantly improved and patient was feeling no more difficulty in breathing. IV analgesics were given (iv pcm and ketorolac). Patient was then able to take deep breaths to achieve lung expansion. Patient maintained spo2 of $95-97 \%$ on venti mask.

\section{Discussion}

Percutaneous nephrolithotomy (PCNL) is a commonly performed, minimally invasive procedure for removal of large size renal and upper ureteric calculi. It is preferred over open technique as it targets the calculus and cause the least trauma to the kidney and adjacent structures. Patient is put in prone position for this procedure. This technique is associated with some technical complications, especially risk of intrathoracic complications is much higher in supracostal approach. ${ }^{1}$

There are different views regarding anesthetic technique to be used for PCNL. The general anesthesia (GA) is being more commonly used for this surgery as compared to regional anaesthesia (RA). However, both GA and RA have been used with their own advantages and disadvantages. GA provides benefit of secure airway in prone position. GA also helps in minimizing injury to the pleura and lungs as controlled tidal volume can be delivered to patient during percutaneous access. Also longer anesthesia duration allows the surgeon to go for multiple and higher punctures if needed. Regional anesthesia (RA) for PCNL was first described in 1988. In spinal anaesthesia, patient experiences less postoperative pain, and early recovery and discharge hence reducing hospital stay. Also blood loss is less in surgery done under regional anaesthesia.

Mehrabi and Shirazi studied the anesthetic and surgical outcomes in patients who had PCNL surgery done under spinal anesthesia in prone position. They found that RA is safe and effective anaesthetic technique for performing PCNL hence shall be considered as a good alternative for GA in adult patients. ${ }^{2}$

A randomized controlled trial of 200 patients was done by H. E. S. Moawad and A. S. El Hefnawy to evaluate the efficiency of GA versus RA in PCNL patients and stated that both groups had almost similar intraoperative hemodynamics. But, postoperative pain scores and use of analgesics were relatively less in the RA group. Patients operated under GA had comparatively more postoperative 
nausea and vomiting but the overall patient satisfaction score was also better. Shivering was more frequent in RA, however the procedures were completed successfully without shifting to GA. ${ }^{3}$

A study by Munver et al evaluated the complications of supracostal approach in PCNL. Supracostal approach was used in 98 patients out of 300 PCNL cases between 19931999. They observed many complications such as haemorrhage at puncture site, haemathorax, hydrothorax, sepsis, nephropleural fistula, pneumothorax, and the incidence of intrathoracic complications in supracostal approach was found to be as high as $23 \%$. In our case report, patient had massive hydrothorax while undergoing PCNL using supracostal approach. ${ }^{4}$

In a similar case report by Munshi et al., hydrothorax could not be diagnosed till the end of surgery because surgery was being done under GA in prone position. The chest auscultation remained normal intraoperatively. At end of surgery they noted the high airway pressures, spontaneous ventilation was achieved. Chest $\mathrm{x}$-ray was done which revealed hydrothorax. Then chest drain was placed and $1500 \mathrm{ml}$ fluid was drained.

In our patient, fortunately we were able to diagnose the hydrothorax during the surgery as our patient had typical symptoms of pleural injury, and moreover patient was under regional anaesthesia so the diagnosis could be made earlier and chest drain was inserted immediately.

Supracostal approach in PCNL operations carry a significant risk of hydrothorax and pneumothorax. Diagnosis is usually delayed because of the position of the patient and General anaesthesia. We believe that regional anaesthesia for PCNL surgeries help in early diagnosis and timely intervention.

\section{Conflict of Interest: None.}

\section{References}

1. S Çakmakkaya, Z Saliho? lu, ? Demiroluk, Y Kose. Unrecognized Massive Hydrothorax during Supracostal Approach for Percutaneous Nephrostolithotomy. Internet J Anesth 2007;16(1).

2. S. Mehrabi and K. K. Shirazi, Results and complications of spinal anaesthesia in PCNL. J Urol, 2010;7:22-5.

3. H. E. S. Moawad and A. S. El Hefnawy. Spinal vs. general anesthesia for percutaneous nephrolithotomy: a prospective randomized trial. Egypt J Anaesth 2015;31(1):71-5.

4. Munver R, Delvecchio FC, Newman GE, Preminger GM. Critical analysis of supracostal access for percutaneous renal surgery. J Surg 2001;166:1242-6.

5. Munshi CA, Bardeen-Henschel A. Hydropneumothorax after percutaneus nephrolithotomy. Anesth Analg 1985;64:840-2.

How to cite this article: Rani A, Kumar P. Massive hydrothorax during supracostal approach for percutaneous nephrolithotomy: A case report. Indian J Clin Anaesth 2019;6(2):302-3. 